\title{
Blocking and reversing hepatic fibrosis in patients with chronic hepatitis B treated by traditional Chinese medicine (tablets of biejia ruangan or RGT): study protocol for a randomized controlled trial
}

\author{
Jianhui Qu ${ }^{1}$, Zujiang Yu ${ }^{4}$, Qin $\mathrm{Li}^{5 \dagger}$, Yongping Chen ${ }^{6 \dagger}$, Dedong Xiang ${ }^{7 \dagger}$, Lin Tan ${ }^{2 \dagger}$, Chunliang $\mathrm{Lei}^{8 \dagger}$, Wenlin Bai ${ }^{1 \dagger}$, \\ Hongyan $\mathrm{Li}^{1+}$, Qinghua Shang ${ }^{3+}$, Liang Chen ${ }^{9}$, Xiaoyu Hu${ }^{10}$, Wei Lu ${ }^{11}$, Zhiqin $\mathrm{Li}^{4}$, Da Chen ${ }^{5}$, Xiaodong Wang ${ }^{6}$, \\ Changjiang Zhang ${ }^{7}$, Guangming Xiao ${ }^{8}$, Xun Qi ${ }^{9}$, Jing Chen ${ }^{10}$, Li Zhou ${ }^{11}$, Guofeng Chen ${ }^{1}$, Yonggang $\mathrm{Li}^{1}$, \\ Zhen Zeng ${ }^{1}$, Guanghua Rong ${ }^{1}$, Zheng Dong ${ }^{1}$, Yan Chen ${ }^{1}$, Min Lou', Chunping Wang ${ }^{1}$, Yinying Lu', \\ Cuihong Zhang ${ }^{1}$ and Yongping Yang ${ }^{1 *}$
}

\begin{abstract}
Background: Chronic hepatitis B (CHB) can progress to cirrhosis, hepatocellular carcinoma (HCC) and ultimately liver-related death. Although oral antiviral therapy for patients with CHB reduces the risk of such complications, once cirrhosis is established, the benefits of antiviral therapy are not robustly demonstrated. According to traditional Chinese medicine (TCM), some Chinese herbal medicines promote blood circulation and soften hard masses, and therefore they may block and reverse hepatic fibrosis. The aim of this study is to evaluate the effects of TCM tablets of the compound biejia ruangan (RGT) administered for fibrosis, and entecavir (ETV), on the development of HCC in patients with CHB or hepatitis B virus (HBV)-related compensated cirrhosis.
\end{abstract}

Methods/design: This multicenter, centrally randomized, double-blind, placebo-controlled, parallel-group study is planned to complete within 5 years. For the study, 1,000 with CHB or HBV-related compensated cirrhosis are randomly assigned in a 1:1 ratio to a treatment group (0.5 mg ETV once daily; $2 \mathrm{~g}$ RGT three times daily) or a control group (0.5 mg ETV once daily; $2 \mathrm{~g}$ RGT dummy agent three times daily). The primary end points are the development of HCC and liver-related death. Secondary end points include disease progression and overall survival.

Discussion: Although antiviral therapy can achieve sustained suppression of HBV replication, thereby preventing cirrhosis, patients with CHB treated with nucleos(t)ide analogs (NUCs) retain a higher risk for HCC compared with patients with inactive disease. Although previous clinical trials with RGT have confirmed the efficacy of blocking and reversing hepatic fibrosis in patients with CHB or compensated cirrhosis, the long-term risk for HCC or disease progression in these patients treated with combination of RGT and NUCs compared with NUCs alone is unclear. Therefore, it is necessary to investigate the effects of the RGT blockade and reversal of hepatic fibrosis on the development of HCC in patients with CHB or HBV-related compensated cirrhosis in large, prospective, multicenter, double-blind, randomized, controlled trials in China. Trial registration: ClinicalTrials.gov Identifier: NCT01965418. Date registered: 17 October 2013

Keywords: Compound biejia ruangan tablet, multicenter randomized controlled trial, chronic hepatitis B, hepatocellular carcinoma, hepatic fibrosis

\footnotetext{
* Correspondence: yongpingyang@hotmail.com

${ }^{\dagger}$ Equal contributors

'Center of Therapeutic Research for Liver Cancer, the 302 hospital of PLA,

100 Xisi Huan Middle Road, Beijing 100039, China

Full list of author information is available at the end of the article
}

\section{() Biomed Central}

(c) 2014 Qu et al.; licensee BioMed Central Ltd. This is an Open Access article distributed under the terms of the Creative Commons Attribution License (http://creativecommons.org/licenses/by/4.0), which permits unrestricted use, distribution, and reproduction in any medium, provided the original work is properly credited. The Creative Commons Public Domain Dedication waiver (http://creativecommons.org/publicdomain/zero/1.0/) applies to the data made available in this article, unless otherwise stated. 


\section{Background}

Hepatitis B virus (HBV) infection is a major health problem worldwide with an estimated 350 million chronic carriers [1], and carriers in China account for $33 \%$ of all chronic carriers globally [2,3]. Although treatment of chronic hepatitis $\mathrm{B}(\mathrm{CHB})$ has dramatically improved over the past decade, chronic HBV infection is still the most common underlying cause of hepatocellular carcinoma ( $\mathrm{HCC}$ ), which remains one of the most common cancers worldwide. A large longitudinal epidemiological study of the natural history of patients with chronic HBV infection has shown that baseline HBV DNA level or cirrhosis is an independent predictor for the development of HCC [4].

Although antiviral therapy can theoretically prevent HCC by inhibiting HBV replication and preventing the development of, or even reversing, cirrhosis, in patients with fibrosis or cirrhosis, eradication or suppression of HBV does not remove this risk and occurrence of HCC, but can control the complications and gain time to prepare for liver transplantation [5-7]. Antiviral therapy has minimal side effects and sustained suppression of HBV replication can be achieved, thus preventing such complications. However, patients with $\mathrm{CHB}$ treated with nucleos (t)ide analogs (NUCs) retain a higher risk for HCC compared with patients with inactive stage disease [8].

According to traditional Chinese medicine (TCM), Chinese medicines promoting blood circulation and softening hard masses may be appropriate for blocking and reversing hepatic fibrosis in $\mathrm{CHB}$ or CHB-related compensated cirrhosis. Over the past decade, the role of TCM in the treatment and prevention of fibrosis has been confirmed by a growing number of experiments and clinical studies [9]. To date, no randomized clinical trial (RCT) has reported whether TCM (tablets of the compound biejia ruangan; RGT) administered to block and reverse hepatic fibrosis in $\mathrm{CHB}$ or HBV-related compensated cirrhosis decreases the risk of developing HCC. RGT (Inner Mongolia Furui Medical Science Co Ltd, Wulanchabu, China) was the first antifibrotic herb approved by the Chinese Food and Drug Administration (CFDA) for fibrotic liver disease in China. The main components of RGT are: turtle shell, zedoray rhizome, peony root, Angelica sinensis, pseudo-ginseng, campanumaea pilosula, Astragalus, dried human placenta, plant worms, Baphicacanthus root and farsythio (Table 1). RGT can soften hardness to dissipate stagnation, and disperse blood stasis and detoxification, and enhance $q i$ (biological substances or activities that preserve life) and blood. Syndromes in Chinese medicine include: (1) weakening of both $q i$ and blood, (2) blockage of meridians (circulation channels for $q i$ ) by blood stasis and (3) generation of dampness and heat (inflammatory pathogens). All composites in this prescription are commonly
Table 1 The major ingredients of fufang biejia ruangan pian (RGT)

\begin{tabular}{lll}
\hline $\begin{array}{l}\text { Chinese phonetic } \\
\text { alphabet name }\end{array}$ & Latin name & English name \\
\hline Biejia & Carapax trionycis & Turtle shell \\
Ezhu & Rhizoma curcumae & Zedoray rhizome \\
Chishao & Radix Paeoniae rubra & Peony root \\
Danggui & Radix Angelica sinensis & Angelica sinensis \\
Sangi & Radix notoginseng & Pseudo-ginseng \\
Dangshen & Radix codonopsis & Campanumaea pilosula \\
Huangai & Radix astragali & Astragalus \\
Ziheche & Placenta hominis & Dried human placenta \\
Dongchongxiacao & Cordyceps sinensis & Plant worms \\
Banlangen & Radix isatidis & Baphicacanthus root \\
Liangiao & Fructus forsythiae & Farsythio \\
\hline
\end{tabular}

used clinically for chronic liver disease, to soften hard masses and dissolve stagnation, and at the same time, to secure essence and eliminate evil. The quality of the herbal drugs was controlled as follows. First, as a specific treatment of liver fibrosis and early cirrhosis, RGT was approved by the CFDA in 1999, with the license number Z1999101. Second, all the components of RGT are purchased from designated and certificated herb plantations. None of these herb providers has changed during the past 10 years. Third, the production workshops for RGT are also certified by the Good Manufacturing Practices standard. No serious adverse effects have been reported even though the drug entered the market $>15$ years ago. Finally, RGT has passed the Good Clinical Practice (GCP) certification by the CFDA, which is the internationally recognized standard for the design, conduct, recording and reporting of clinical trials involving human subjects.

Some clinical trials have demonstrated that RGT can block the development of hepatic fibrosis and reverse early cirrhosis [10]. Other studies have shown that RGT can significantly improve compensated cirrhosis, especially in $\mathrm{CHB}$ and early cirrhosis, with a total rate of effectiveness of $81.67 \%$; after a 1-year follow-up, the total rate of efficacy was shown to be $76.85 \%$ [11]. Yang et al. [12-14] have reported that RGT significantly blocks early liver fibrosis, inhibiting the proliferation of hepatic stellate cells, reducing collagen synthesis and excess deposition in the space of Disse, dissolving and absorbing formed collagen fibers, and inhibiting expression of collagen $\alpha 2$ (I) mRNA. It has also been shown that RGT increases the phagocytic function of macrophages in the abdominal cavity of mice. Zhao et al. [15] have reported similar results, and have proposed that RGT has antifibrotic efficacy through interaction with multiple targets that affect hepatic fibrosis. Some studies 
have confirmed that RGT has better antifibrotic efficacy in compensated cirrhosis [16,17], especially HBV-related hepatic fibrosis [18].

Although some studies have concluded that RGT can block and reverse hepatic fibrosis in $\mathrm{CHB}$, there are limited data on preventing or delaying the development of $\mathrm{HCC}$ in the treatment of CHB or HBV-related compensated cirrhosis. To address this issue, we are conducting a large, prospective, multicenter, centrally randomized, double-blind, controlled trial to establish whether RGT can prevent or delay the development of HCC by blocking and reversing hepatic fibrosis in $\mathrm{CHB}$ or $\mathrm{HBV}$ related compensated cirrhosis. The aims of this clinical trial are: (1) to evaluate the effect of combined application of RGT and entecavir (ETV) on development of HCC in patients with $\mathrm{CHB}$ or HBV-related compensated cirrhosis and (2) to evaluate the efficacy of combined application of RGT and ETV in preventing or delaying the incidence of decompensated cirrhosis and disease progression in the treatment of patients with $\mathrm{CHB}$ or HBVrelated compensated cirrhosis.

\section{Methods/design Study design}

This large, prospective, multicenter, centrally randomized, double-blind, placebo-controlled trial is being conducted in nine institutions between November 2013 and November 2018. The study meets the requirements of the Declaration of Helsinki, and has been approved by the ethics committees of each participating institution. All patients with $\mathrm{CHB}$ or HBV-related compensated cirrhosis are carefully screened by a multidisciplinary team to select suitable candidates for this clinical trial, according to the inclusion and exclusion criteria of the present RCT and the patients' willingness to participate in the study. For the study, 1,000 patients are being randomly assigned in a 1:1 ratio to one of two groups: a treatment group (0.5 mg ETV once daily; 2 g RGT three times daily) or a control group [0.5 mg ETV once daily; $2 \mathrm{~g}$ RGT dummy agent (similar in taste, shape and color to RGT; main ingredients include pearl barley and grilled germinate barley) three times daily]. During the doubleblind phase, unblinding will take place as soon as sufficient evidence indicates: (1) that the effect of RGT plus ETV blocking and reversing hepatic fibrosis (measured by METAVIR system) is statistically superior to that with ETV alone, (2) that RGT plus ETV does not provide a significant advantage over ETV alone by the second liver biopsy at week 72 of double-blind treatment or (3) safety concerns. Patients who reach an end point are offered open-label RGT plus ETV or ETV alone for 1 year, and patients who have blockade and reversal of hepatic fibrosis are followed up after treatment and have the option to receive RGT plus ETV as an open-label treatment in the event of hepatic fibrosis or necroinflammatory progression. However, patients with stable or reversed disease will continue to receive antiviral therapy with ETV alone, but those with disease progression will receive open-label RGT plus ETV as in the control group. The study will be terminated as soon as sufficient evidence indicates that the outcome of patients with $\mathrm{CHB}$ or HBV-related compensated cirrhosis by RGT plus ETV blockade and reversal of hepatic fibrosis is statistically superior to that with ETV alone or does not provide a significant advantage over ETV. If the trial is terminated according to the predefined criteria, patients will be offered open-label treatment for 1 year. Liver biopsies are performed at three defined time points: before the start of randomization (pretreatment), at week 72 of the double-blind treatment, and at month 24 of the open-label treatment in the follow-up study. After 24 weeks of treatment, any patients with a reduction in HBV DNA level from baseline $<1$ log IU or $2 \log$ copies/mL will be treated with a combination of ETV and adefovir dipivoxil (10 mg once daily) against HBV. The flow chart of the study is shown in Figure 1.

This study is being conducted in accordance with the ethical principles of the Declaration of Helsinki and is consistent with GCP guidelines and applicable local regulatory requirements. Written informed consent is obtained from all randomly assigned patients.

The primary end points in this study are the occurrence of HCC or liver-related mortality. HCC is diagnosed either histologically or clinically based on guidelines proposed by the Chinese Liver Cancer Study Group [19]. According to these criteria, patients are considered positive for HCC if they have one or more risk factors (hepatitis $\mathrm{B}$ or $\mathrm{C}$ virus infection, or cirrhosis) and one of the following: (1) serum $\alpha$-fetoprotein (AFP) $>400 \mathrm{ng} / \mathrm{mL}$ and a positive finding on at least one of three typical imaging studies [dynamic computed tomography (CT), dynamic magnetic resonance imaging (MRI) or hepatic angiography] or (2) serum AFP $<400 \mathrm{ng} / \mathrm{mL}$ and positive findings on at least two of the three imaging studies. A positive finding for typical HCC on dynamic CT or MRI is defined as increased arterial enhancement followed by decreased enhancement compared with the liver (washout) in the portal or equilibrium phase. Liver-related mortality is defined as death caused by hepatic decompensation (regardless of etiology) or HCC.

Secondary end points are disease progression and overall survival rate. Disease progression is defined as either histological progression reflected by an increase in METAVIR score of at least 1 point or clinical progression reflected by the occurrence of decompensated cirrhosis. Decompensated cirrhosis is defined by the development of complications of portal hypertension (ascites, variceal hemorrhage, hepatic encephalopathy or spontaneous bacterial peritonitis) and/or liver insufficiency (jaundice). 


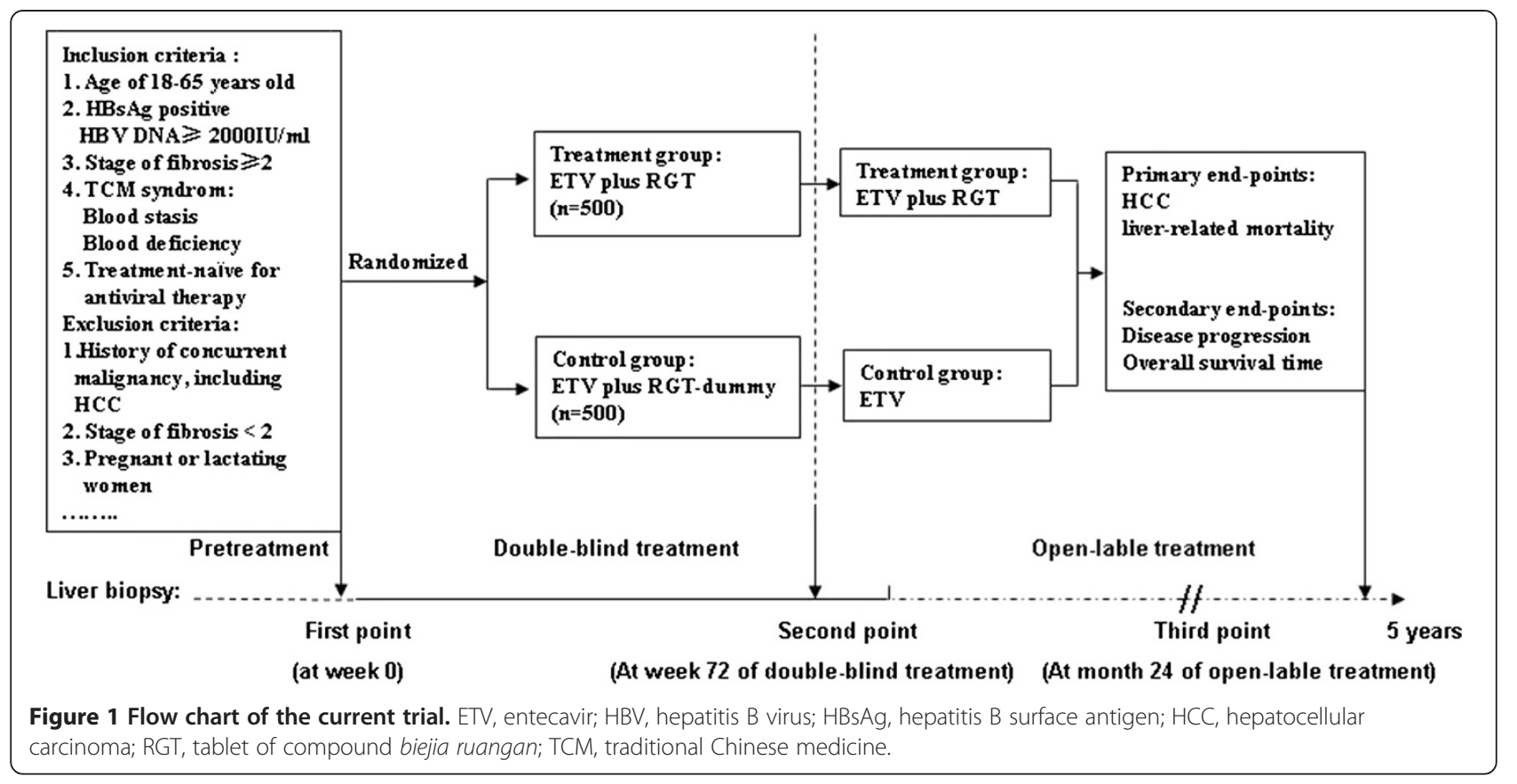

Following previous studies [20,21], liver biopsies are collected at three defined time points: before the start of randomization (unless a biopsy has been performed within 3 months of randomization), at week 72 of the doubleblind treatment and at month 24 of the open-label treatment during follow-up. Every patient with liver biopsy of at least six portal tracts is included in the study. To ensure the quality of liver tissue specimens for pathological assessment, liver biopsies in all centers is performed using unified 16G liver biopsy needles and a modified Jamshidi Menghini needle (Allegiance Corporation, McGaw Park, IL, USA), and the tissue is fixed in formalin and embedded in paraffin. Two independent third-party pathologists who are unaware of the patients' treatment assignment, biopsy sequence and clinical outcome will examine all biopsy slides.

Hepatic fibrosis is assessed by the METAVIR system for fibrosis stage and necroinflammatory activity as a hard index [22,23]. The fibrosis stages are: no fibrosis (F0), mild fibrosis (F1), moderate fibrosis (F2), severe fibrosis (F3) and cirrhosis (F4). Necroinflammatory activity is: no activity (A0) mild activity (A1), moderate activity (A2) and severe activity (A3). Serum fibrosis biomarkers including Fibrotest, aspartate aminotransferase to the platelet ratio index (APRI), liver stiffness spleen diameter to platelet ratio score (LSPS), and FibroMeters and FibroScan are used to assess hepatic fibrosis as a surrogate index $[24,25]$. Hard and surrogates indexes are evaluated at all three time points (baseline, week 72 and week 96 after the open label) for each patient. Changes in liver fibrosis from baseline to weeks 72 and weeks 96 after the open label are analyzed on available pooled data using the METAVIR scoring system and the surrogate index.

The contraindications of liver biopsy include coagulopathy, uncooperative patients, impaired mental status, infection of the hepatic bed and extrahepatic biliary obstruction. In addition, patients undergoing a liver biopsy are required to have an international normalized ratio $<1.6$ and platelet count $>60 \times 10^{9} / \mathrm{L}$. For analysis of the histological end point, patients who can be evaluated must have adequate baseline biopsy specimens with a METAVIR necroinflammatory or fibrosis score of at least 1. Progression of hepatic fibrosis or necroinflammatory progression is defined as an increase of at least 1 point.

\section{Follow-up}

At baseline, all patients are screened to confirm the absence of HCC using abdominal ultrasonography and laboratory analyses, including AFP level. During the follow-up, all patients are seen at 3- to 6-month intervals. Periodic surveillance is also performed with abdominal ultrasonography and laboratory analyses, including AFP level, every 3 or 6 months to screen for HCC and other complications related to portal hypertension. Patients also undergo endoscopy every 1 or 2 years for screening and follow-up of gastroesophageal varices. HBV DNA, hepatitis B surface antigen (HBsAg), antiHBs, hepatitis $B$ e antigen, anti-HBe, and routine hematological and biochemical tests are performed. Adverse events that have occurred since the previous visit are recorded, and toxicity is graded according to predefined criteria. 


\section{Participants and eligibility}

Inclusion criteria are as follows: (1) men or women aged 18 to 65 years; (2) persistent serum $\mathrm{HBsAg}$ for $\geq 6$ months; (3) serum HBV DNA $\geq 2000 \mathrm{IU} / \mathrm{mL}$ or $\geq 10,000$ copies/mL; (4) baseline liver fibrosis (liver biopsy) stage F $\geq 2$ (METAVIR system); (5) TCM syndrome type: blood stasis, or blood deficiency with toxic heat retention and (6) no antiviral or antifibrotic therapy for 6 months. Patients are excluded if they have any of the following at enrollment: (1) history of concurrent malignancy, including HCC; (2) baseline liver fibrosis (liver biopsy) stage F <2 (METAVIR system); (3) immunosuppression (organ transplantation or immunosuppressant use); (4) severe alcoholism; (5) serious uncontrollable heart, kidney, lung, endocrine, blood, metabolic or gastrointestinal primary disease, or mental illness; (6) pregnancy or lactation; (7) allergic constitution or allergic to TCM use; (8) no prescribed medication, poor compliance, incomplete data affecting the efficacy and safety of those judgments; (9) unsuitable for this trial according to the researchers or (10) co-infection with hepatitis $\mathrm{C}$ or $\mathrm{D}$ virus or HIV.

\section{Recruitment}

Nine first-class hospitals across the Eastern, Western, Southern, Northern and Central areas of China are involved in this clinical trial. Participants are recruited via posters at each participating center.

\section{Randomization}

This study has two arms. Once enrolled, participants who meet the inclusion criteria are informed about the study and treatment plan. Written informed consent is obtained when the patient accepts this plan and is willing to participate. A consecutively numbered envelope is unsealed by a research assistant. The sealed numbers are generated by a computerized random number generator with SAS software by Boao Tong Medical Technology Co Ltd (Beijing, China) and are concealed and disseminated using opaque envelopes. Based on this number, the patient is randomly assigned in a $1: 1$ ratio to the treatment group (ETV + RGT, $n=500$ ) or control group (ETV + RGT-dummy agent, $n=500)$.

\section{Sample size}

We assumed that the rate of hepatic fibrosis reversal would be $39 \%$ in the ETV-treated patients at the end of 2 years [26,27], and $61 \%$ in the RGT-treated patients. Using PASS 2002 statistical software (NCSS, Kaysville, UT, USA), we calculated that we need two groups of 186 cases, and each group needs to enroll 223 cases if there is a drop-out rate of $15 \%$, that is, a total of 446 cases. Currently, up to $45 \%$ of all enrolled patients will be accepted for a second liver biopsy. A total of 500 cases is required in each group to achieve a power of 0.80 and a significance level of 0.05 in assessing the difference between the two groups.

\section{Safety}

All adverse events, regardless of their possible association with the disease or study treatment, are recorded. Adverse events are considered to be serious if the investigator determines that they jeopardize the patient, are life-threatening, or could result in hospitalization, disability or death.

\section{Data and safety monitoring board}

To promote consistent study execution at each participating center, independent monitoring visits are performed to supervise trial progress, ensuring that it is conducted, recorded and reported in accordance with the protocol, standard operating procedures, GCP and the applicable regulatory requirements.

The Data and Safety Monitoring Board consists of three independent hepatologists who are not members of the end-point committee and an independent statistician. The board protects the ethical interests and safety of the patients by reviewing interim analyses. The board is empowered to recommend termination of the study on the basis of safety concerns or as soon as sufficient evidence indicates that RGT plus ETV is statistically superior to ETV alone, or that RGT plus ETV does not provide a significant advantage over ETV alone.

\section{Ethics}

The study protocol, written informed consent and posters were approved by the 302nd Military Hospital Ethics Review Committee (2013145D), and were accepted by the ethics committees at each of the institutions involved, and have been performed according to the Declaration of Helsinki.

The names of all ethical bodies in the various centers involved are the 302nd Military Hospital Ethics Review Committee, Fuyang No 2 People's Hospital Ethics Review Committee, 88th Hospital of PLA Ethics Review Committee, First Affiliated Hospital of Zhengzhou University Ethics Review Committee, Fuzhou Infectious Diseases Hospital Ethics Review Committee, First Affiliated Hospital of Wenzhou Medical University Ethics Review Committee, First Affiliated Hospital of Third Military Medical University Ethics Review Committee, Guangzhou No 8 People's Hospital Ethics Review Committee, Shanghai Public Health Clinical Center Ethics Review Committee, Affiliated Hospital of Chengdu University of Traditional Chinese Medicine Ethics Review Committee and Tianjin Second People's Hospital Ethics Review Committee. 


\section{Statistical methods}

The randomized subjects who have taken at least one dose of study drug and are evaluated for at least once curative effect after taking medication, constitute the full analysis set and safety analysis set. Those who complete all the visits comprise the per-protocol set. The statistical analysis will be performed using SAS 9.1 software (SAS Institute, Cary, NC, USA). The overall loss rate due to adverse events of each group will be calculated. When subjects enter the trial, they are assessed for demographic data, baseline condition and other essential information and are compared between groups. Cumulative incidence of $\mathrm{HCC}$ and overall survival rate will be determined by Kaplan-Meier analysis, and the log-rank test will be used for treatment comparisons. Changes from baseline in liver histology (METAVIR score) as well as in liver and spleen imaging (type $B$ ultrasound) between the two groups will be compared by the Cochran-Mantel-Haenszel test. Changes from baseline in serum markers for liver fibrosis and liver function, transient electrograph (FibroScan) and TCM syndrome between the two groups will be compared by a covariance or non-parametric test. The statistical significance is defined as a two-sided $P<0.05$.

\section{Discussion}

$\mathrm{HBV}$ is a common infection that is associated with a considerable burden of liver-related morbidity and mortality worldwide [28]. HBV is strongly associated with HCC by its presence in tumor cells and by the role of persistent HBV infection as a risk factor for the development of HCC [29]. Recent advances have seen the introduction of drug therapy with the potential to have a significant impact on the incidence of liver-related complications [28]. In particular, treatment of $\mathrm{CHB}$ has dramatically improved over the past decade [30-32], but persistent $\mathrm{HBV}$ infection is still the most common underlying cause of $\mathrm{HCC}$, which remains one of the most common cancers worldwide [33]. Cirrhosis and persistently high viremia are both important risk factors for $\mathrm{HCC}$ in patients with $\mathrm{CHB}[4,33,34]$. Therefore, antiviral therapy could theoretically prevent $\mathrm{HCC}$ by inhibiting HBV replication and preventing the development of, or even reversing, cirrhosis [30-32]. Antiviral therapy alone does not remove this risk and occurrence of HCC [7]. However, it has not been demonstrated whether a combination of TCM and antiviral therapy could have a role in preventing or delaying the development of $\mathrm{HCC}$ in patients with $\mathrm{CHB}$.

Clinical trials have been carried out to evaluate the role and efficacy of RGT for HBV-related hepatic fibrosis and compensated cirrhosis [10,15-18]. RGT administered to block and reverse hepatic fibrosis in $\mathrm{CHB}$ or $\mathrm{HBV}$ related compensated cirrhosis is considered one of the treatment options for patients with hepatic fibrosis. Only limited data are available about the effects of RGT blockade and reversal of hepatic fibrosis on the development of HCC in patients with $\mathrm{CHB}$. To date, there have been no RCTs comparing the effects of combined application of RGT and NUCs with NUCs alone on the development of $\mathrm{HCC}$ in patients with $\mathrm{CHB}$ or $\mathrm{HBV}$-related compensated cirrhosis.

The present clinical trial introduces promising and classical herbs and formulae into the treatment of hepatitis-B-related hepatic fibrosis and compensated cirrhosis. Above all, this clinical study evaluates for patients with $\mathrm{CHB}$ or HBV-related compensated cirrhosis: (1) the efficacy of TCM (RGT) in preventing or delaying the occurrence of HCC, (2) the efficacy of RGT in reducing the development of decompensated cirrhosis and disease progression for patients with $\mathrm{CHB}$ or HBV-related compensated cirrhosis and (3) whether TCM treatment improves the hepatic microenvironment and reduces the development of HCC.

In summary, the results of the present trial are of value because they will provide an important evidence base for the effects of TCM blockade and reversal of hepatic fibrosis on development of $\mathrm{HCC}$ for patients with $\mathrm{CHB}$ or HBV-related compensated cirrhosis.

\section{Trial status}

Patient recruitment for this trial is ongoing. Data collection will continue until the end of November 2018.

\section{Abbreviations}

AFP: a-fetoprotein; APRI: aspartate aminotransferase to the platelet ratio index; CFDA: Chinese Food and Drug Administration; CHB: chronic hepatitis B; CT: computed tomography; ETV: entecavir; GCP: Good Clinical Practice; HBsAg: hepatitis B surface antigen; HBV: hepatitis B virus; HCC: hepatocellular carcinoma; LSPS: liver stiffness spleen diameter to platelet ratio score; MRI: magnetic resonance imaging; NUC: nucleos(t)ide analog; $\mathrm{RCT}$ : randomized clinical trial; RGT: tablet of compound biejia ruangan; TCM: traditional Chinese medicine.

\section{Competing interests}

The authors declare that they have no competing interests.

\section{Authors' contributions}

YY conceived and designed the clinical trial, recruited patients, and wrote and gave final approval for the manuscript. JQ conceived and designed the clinical trial, recruited patients, contributed reagents and materials, and gave final approval for the manuscript. HL performed the clinical trial, contributed reagents and materials, wrote and gave final approval for the manuscript. $Z Y$, $Q L, L T, W B$ and $Q S$ recruited patients, performed the clinical trial, and gave final approval for the manuscript. YC, DX, CL, LC, XH, WL, ZL, DC, XW, CZ, GX, $X Q, J C, L Z, G C, Y L, Z Z, M L$ and $C W$ recruited patients and gave final approval for the manuscript. ZD, GR and YC recruited patients, performed the clinical trial, contributed reagents and materials, and gave final approval for the manuscript. YL recruited patients, contributed reagents and materials, and gave final approval for the manuscript. CZ performed the clinical trial, contributed reagents and materials, and gave final approval for the manuscript. All authors read and approved the final manuscript.

\section{Acknowledgements}

The present study is supported as a National Science and Technology Major Project (2013ZX10005002) 


\section{Author details}

${ }^{1}$ Center of Therapeutic Research for Liver Cancer, the 302 hospital of PLA, 100 Xisi Huan Middle Road, Beijing 100039, China. ${ }^{2}$ Liver Disease Department, Fuyang No 2 People's Hospital, Fuyang, Anhui Province 236015, China. ${ }^{3}$ Therapeutic Center for Liver Disease, 88th Hospital of PLA, Taian, Shandong Province 271000, China. ${ }^{4}$ Department of Infectious Disease, First Affiliated Hospital of Zhengzhou University, Zhengzhou, Henan Province 450052, China. ${ }^{5}$ Fuzhou Infectious Diseases Hospital, Fuzhou, Fujian Province 350025, China. ${ }^{6}$ Department of Infectious and Liver Diseases, Liver Research Center, First Affiliated Hospital of Wenzhou Medical University, Wenzhou, Zhejiang Province 325000, China. ${ }^{7}$ Department of Infectious Diseases, Southwest Hospital, Third Military Medical University, Chongqing 400038, China. ${ }^{8}$ Guangzhou No 8 People's Hospital, Guangzhou, Guangdong Province 510060, China. ${ }^{9}$ Department of Hepatic Diseases, Shanghai Public Health Clinical Center, Shanghai 201508, China. ${ }^{10}$ National Integrative Medicine Clinical Base for Infectious Diseases, Department of Infectious Diseases, Affiliated Hospital of Chengdu University of Traditional Chinese Medicine, Chengdu, Sichuan Province 610072, China. ${ }^{11}$ Tianjin Second People's Hospital, Tianjin Institute of Hepatology, Tianjin 300192, China.

\section{Received: 31 July 2014 Accepted: 24 October 2014}

Published: 10 November 2014

\section{References}

1. Lavanchy D: Hepatitis B virus epidemiology, disease burden, treatment, and current and emerging prevention and control measures. J Viral Hepatitis 2004, 11:97-107.

2. Zhao H, Si CW: Chinese epidemic status of viral hepatitis B. Chin J Front Med Sci 2008, 1:1-3.

3. Zhang C, Zhong Y, Guo L: Strategies to prevent hepatitis B virus infection in China: immunization, screening, and standard medical practices. BiosCi Trends 2013, 7:7-12.

4. Chen CJ, Yang HI, Su J, Jen CL, You SL, Lu SN, Huang GT, Iloeje UH, REVEALHBV Study Group: Risk of hepatocellular carcinoma across a biological gradient of serum hepatitis B virus DNA level. JAMA 2006, 295:65-73.

5. Fink SA, Jacobson IM: Managing patients with hepatitis-B related or hepatitis-C-related decompensated cirrhosis. Nat Rev Gastroenterol Hepatol 2011, 8:285-295.

6. Chen LP, Zhao J, Du Y, Han YF, Su T, Zhang HW, Cao GW: Antiviral treatment to prevent chronic hepatitis B or C-related hepatocellular carcinoma. World J Virol 2012, 1:174-183.

7. Papatheodoridis GV, Manolakopoulos S, Touloumi G, Vourli G, RaptopoulouGigi M, Vafiadis-Zoumbouli I, Vasiliadis T, Mimidis K, Gogos C, Ketikoglou I: Virological suppression does not prevent the development of hepatocellular carcinoma in HBeAg-negative chronic hepatitis B patients with cirrhosis receiving oral antiviral(s) starting with lamivudine monotherapy: results of the nationwide HEPNET. Greece cohort study. Gut 2011, 60:1109-1116.

8. Cho JY, Paik YH, Sohn W, Cho HC, Gwak GY, Choi MS, Lee JH, Koh KC, Paik SW, Yoo BC: Patients with chronic hepatitis B treated with oral antiviral therapy retain a higher risk for HCC compared with patients with inactive stage disease. Gut. in press.

9. Chen $\mathrm{Q}, \mathrm{Wu} \mathrm{ZL}$, Zhong $\mathrm{H}$ : Study of treatment of anti-fibrosis compound prescription of Traditional Chinese Medicine. Chin J Integrated Traditional Western Med Liver Diseases 2004, 14:252-254.

10. Miao WH: Compound Biejia Ruangan tablet treatment clinical observation of 40 patients with liver cirrhosis. Mod Med 2009, 15:140.

11. Chen JM, Yang YP, Chen DY: Compound Biejia Ruangan tablet clinical study on the treatment of chronic hepatitis B with hepatic fibrosis. Chin J Exper Clin Virol 2007, 21:358-360.

12. Yang YP, Song XX, Chen DY, Chen JM, Zhang B: The regulation function of $Y$-IFN, IL-2, IL-6 and tocopherol for proliferation of hepatic stellate-cells and synthesizing collagen on primary cultured hepatic stellate-cells. Chin Clin Hepatol 1995, 11:72-75.

13. Yang YP, Song XX, Chen DY, Zhu AJ, Zhang B, Sheng CJ: Effects of Traditional Chinese Medicine compound Biejia Ruangan tablet on $\mathrm{CCl}_{4}$ induced cirrhotic rats. Western Traditional Med Integration J Hepatol 1994 4:22-24

14. Yang YP, Chen DY, Song XX, Zhu AJ, Zhou YX: Effects of Traditional Chinese Medicine compound Biejia Ruangan tablet on the proliferation of hepatic stellate-cells and expression of collagen type I or Ш mRNA. Western Traditional Med Integration J Hepatol 1995, 5:26-28.
15. Zhao JM: Anti-hepatic fibrosis mechanisms of compound Biejia Ruangan tablet. Med J Chin People's Liberation Army 2004, 29:560-562.

16. Hao HS, Li G: Early clinical application and observation of Fufang Biejia Ruangan tablet on the treatment of liver fibrosis. Med Innovation Chin 2009, 6:36

17. Geng YM: Fufang Biejia Ruangan tablet on cirrhosis treatments: clinical observation of 60 cases. Chin Healthcare Innovation 2009, 4:31.

18. $\mathrm{Xu} \mathrm{QH}$ : Observations on anti-hepatic fibrosis effect of compound Biejia Ruangan tablet. Chin J Ethnomed Ethnopharm 2009, 18:72.

19. Chen MS, Chen MH, Ye SL, Qin SQ: Expert consensus on the ablative therapies for hepatocellular carcinoma. Chin Clin Oncol 2011, 16:70-73.

20. Chang TT, Gish RG, Man RD, Gadano A, Sollano J, Chao YC, Lok AS, Han KH, Goodman Z, Zhu J, Cross A, DeHertogh D, Wilber R, Colonno R, Apelian D: A comparison of entecavir and lamivudine for $\mathrm{HBeAg}$-positive chronic hepatitis B. N Engl J Med 2006, 354:1001-1010.

21. Dienstag JL, Goldin RD, Heathcote EJ, Hann HWL, Woessner M, Stephenson SL, Stephen G, Gray DF, SCHIFF E: Histological outcome during long-term lamivudine therapy. Gastroenterology 2003, 124:105-117.

22. Poynard T, Bedossa P, Opolon P: Natural history of liver fibrosis progression in patients with chronic hepatitis C. Lancet 1997, 349:825-832.

23. Poynard T, McHutchison J, Manns M, Trepo C, Lindsay K, Goodman Z, Ling $\mathrm{MH}$, Albrecht J: Impact of pegylated interferon alfa-2b and ribavirin on liver fibrosis in patients with chronic hepatitis C. Gastroenterology 2002, 122:1303-1313.

24. Rockey DC, Bissell DM: Noninvasive measures of liver fibrosis. Hepatology 2006, 43(Suppl 1):S113-S120

25. Martinez SM, Crespo G, Navasa M, Forns X: Noninvasive assessment of liver fibrosis. Hepatology 2011, 53:325-335.

26. Chang TT, Lai CL, Yoon SK, Lee SS, Coelho HSM, Carrilho FJ, Poordad F, Halota W, Horsmans Y, Tsai N, Zhang H, Tenney DJ, Tamez R, lloeje U: Entecavir treatment for up to 5 years in patients with hepatitis $\mathrm{B}$ e antigen-positive chronic hepatitis B. Hepatology 2010, 51:422-430.

27. Jeng WJ, Sheen IS, Chen YC, Hsu CW, Chien RN, Chu CM, Liaw YF: Off-therapy durability of response to entecavir therapy in hepatitis $B$ e antigennegative chronic hepatitis B patients. Hepatology 2013, 58:1888-1896.

28. Aspinall EJ, Hawkins G, Fraser A, Hutchinson SJ, Goldberg D: Hepatitis B prevention, diagnosis, treatment and care: a review. Occup Med 2011, 61:531-540.

29. Park $\mathrm{NH}$, Song $\mathrm{IH}$, Chung $\mathrm{YH}$ : Chronic hepatitis B in hepatocarcinogenesis. Postgrad Med J 2006, 82:507-515.

30. Lok ASF, McMahon BJ: Chronic hepatitis B. Hepatology 2007, 45:507-539.

31. Papatheodoridis GV, Manolakopoulos S, Dusheiko G: Therapeutic strategies in the management of patients with chronic hepatitis B. Lancet Infect Dis 2008, 8:167-178.

32. European Association for the Study of the Liver: EASL clinical practice guidelines: management of chronic hepatitis B. J Hepatol 2009, 50:227-242.

33. El-Serag HB, Rudolph KL: Hepatocellular carcinoma: epidemiology and molecular carcinogenesis. Gastroenterology 2007, 132:2557-2576.

34. Schafer DF, Sorrell MF: Hepatocellular carcinoma. Lancet 1999, 353:1253-1257.

\section{doi:10.1186/1745-6215-15-438}

Cite this article as: Qu et al.: Blocking and reversing hepatic fibrosis in patients with chronic hepatitis B treated by traditional Chinese medicine (tablets of biejia ruangan or RGT): study protocol for a randomized controlled trial. Trials 2014 15:438.

\section{Submit your next manuscript to BioMed Central and take full advantage of:}

- Convenient online submission

- Thorough peer review

- No space constraints or color figure charges

- Immediate publication on acceptance

- Inclusion in PubMed, CAS, Scopus and Google Scholar

- Research which is freely available for redistribution 